Oceans - Europe 2005

\title{
Ships Confining an Oil Spill Over: A Scenario for Automatized Cooperation
}

\author{
J.F. Jimenez, J.M. Giron-Sierra, A. Dominguez, \\ J.M. De la Cruz, and J.M. Riola ${ }^{(1)}$ \\ Dep. ACYA, Fac. CC. Fisicas, Universidad Complutense de Madrid \\ Ciudad Universitaria \\ 28040 Madrid, Spain. \\ gironsi@dacya.ucm.es \\ (1) Canal de Experiencias Hidrodinamicas. CEHIPAR \\ El Pardo. Madrid, Spain
}

\begin{abstract}
Cooperation between robots is an important contemporary issue. This can be translated to the marine environment, elther using marine robots or introducing automatics in the operations of ships. A general research on this kind of problems has started in our group, after several years of developing autonomous robotized ships. Several scenarios have been proposed for the study of cooperation details. This paper focus in a very interesting case, which is representative of other cases: several ships towing booms for oil spill over confinement. It turns out that the cooperation problem is not trivial. Along the operation several phases can be distinguished, and several coordination problems and needs appear. A computer simulation has been developed, after physics based analysis, and some initial coordinated control strategies have been proposed and tested. These strategies are supposed to be applied through verbal orders to captains. Along the operation phases the role of captains change, for an adaptive coordination. The paper introduces the research topic, then describes the scenario and its simulation, then focus on the cooperation problems emerging from the operation phases and the control and coordination solutions that bave been proposed, and finally draws some conclusions.
\end{abstract}

\section{INTRODUCTION}

After seven years of research on seakeeping control of ships by moving actuators such flaps and T-foils $[1,2]$, a widet research on automatized cooperation of marine crafts has been launched. There are important reasons in favour of this initiative. Also, recent ecological catastrophes, like the Prestige sinking near the Spain coast, have motivated a particular attention to marine operations involving several ships and submergibles, to avoid as much as possible the effects of oil spill over.

During the last part of our research on seakeeping control, a six degrees of freedom approach has been followed. This was a continuation of the first studies in a towing tank with bead seas. A scaled down replica of a fast ferry has been built. This replica has on-board autonomous control, including speed, heading and seakeeping control. The replica has self-knowledge of its position.

Since our field is automatic control and robotics, it was clear for us that the autonomous scaled ship is a kind of surface marine robot. Moreover, since part of our research concerns teams of mobile robots, the idea of taking the sea as an scenario of automatized cooperation between robots naturally arised.

Indeed, the daily contact with people having long experience in the sea, gave us the impression that a good realistic alternative could be to include computers on board the cooperating ships, exchanging verbal messages and taking decisions under the supervision of the ships captains. A communication protocol should be established for such purpose.

This research started by considering some different scenarios requiring cooperation of ships. First studies began with animated simulations, helping us to capture the specific needs along the operations, for a good cooperative work. In paralell a set of scaled ships are under construction, for experimental studies in the same scenarios considered by the simulations.

This paper focuses on an interesting scenario. The general case will be several ships towing a boom, for oil spill over confinement. The study began with the simplest cooperation case: two ships towing the boom.

The order in this paper will be the following. The next two sections will be devoted to a condensed review of cooperation in tobot teams, and its translation to the marine environment. Then, the oil spill over confinement case will be studied. Then, physics based modeling will be introduced and the simulation will be briefly described. Finally, the operation will be studied, showing the specific cooperation needs that appear. The paper finish with some conclusions in view of the practical development of protocols.

\section{ROBOTS AND COOPERATION}

The initial research on mobile tobots proposed hierarchical behaviour control architectures. A representative metaphor for the on-board control was a three person structure. A captain deciding where to go and the main traits of the path. Then, there is an officer to elicitate more planning details. And there is a pilot governing in real-time the ship, executing with some adaptations the plan. It was found that the hierarchical structure may lead to rigid and complicated control. A reactive paradigm was proposed in the form of a subsumption architecture [3], which offers a simpler way for robot behaviour building. However, the general problems of baving a target and deciding how to reach it, requires some deliberative features. As [4] asserts, the consensus is now to combine deliberative and reactive paradigms.

There are two main axes for cooperation in a robot team. One axis is distribution of responsibilities. The other axis is time. There are coordination needs, and perbaps syncronization could also be needed. The book [5] provides examples of several terrestrial mobile robots pushing together and object, and other multiple physical agent cases with leaming, adaptation, self-organisation and spatial distribution aspects. Protocols for robotic agents communication and interaction are more specifically studied in [6] This reference says that the tasks must be assigned to agents along 
time in function of the expertise and aptitude (it includes physical position) of the agents in each moment. The planning and decisions could be done in a centralized or distributed way. A blackboard may be used for the team decisions.

There are two main extremes. One is to bring full authority to a central coordinator. The other is all agents having full autonomy. There are intermediate formulas, combining central authority with some delegation in a certain degree. This degree may vary along an operation. Related with this is the issue of the combination of local knowledge and general knowledge about the operational status that agents should have. Part of our specific experience, in a distributed avionic control system, is related with this matter [7]. In this case, smart components usually decide in function of local knowledge (for instance, a valve closing when a particular tank reaches a specified level), but they may have a different behaviour if they receive a message telling there is a fault mode in the system.

Computer networking also leads to cooperation studies and developments. The book [8] on computer supported cooperative work includes interesting pages on multiagent systems, shated information, voting decision schemes, conflicts and reconfiguration, negotiation and contracts between agents, etc. Figure 1 reproduces the $3 \mathrm{C}$ model in [8], embodying communication, coordination and cooperation. The communication support consists in message systems. The cooperation support includes conversation contents and protocols. The coordination support manages the workflow carried out by the team. Figure 2 focus on the time axis, and the kind of know-how that may be called upon.

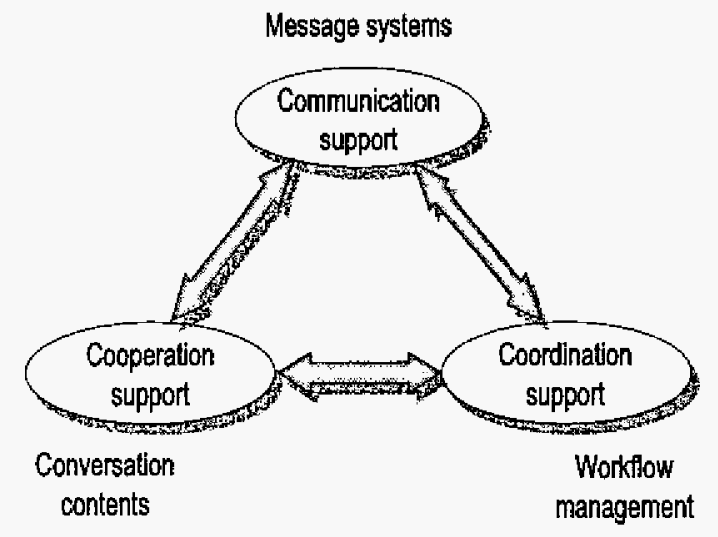

Fig. 1. 3C model : communication, coordination and cooperation.



Fig. 2. Cooperation issues felated to time.
An interesting contemporary topic, which is relevant for our research, is formation control. Reference [9] on following-the-leader is a good recent source of information on the topic.

\section{COOPERATION IN MARITIME SCENARIOS}

There are many maritime circumstances or operations involving several marine crafts. For instance, the maneuvering of ships in confined waters or channels, to avoid collisions. In certain cases there are attracting forces that cause difficulties [10]. This is also the case of freight or person offshore transfers between two ships in motion. This example can be seen as a particular instance of a general rendez-vous operation category, which can be static or in motion. Some recent papers on this kind of problems are [11],[12] and [13].

The deployment of nets, sets of buoys, barriers, etc. is another kind of operation that usually requires cooperation of ships. A particular case is the confinement of oil spill overs. This operation may continue with recycling, towing, suction, etc.

Other scenarios may include some sort of formation control, not only in the case of military or fishing operations. For instance, high speed transportation may require a temporary corridor to be respected by other ships.

Emergency or disaster cases may involve special cooperative towing tasks, scanning for survivors in a certain zone using a team of ships and aircrafts, etc. Patrol and security operations can be of similar nature. This is connected with robotic topics about exploration and harvesting by, perhaps heterogeneous, robot teams.

Recent scientific meetings are beginning to devote sessions to robot coordination in a marine context, with papers such [14] about coordinated motion control, or [15] on towing.

The interest for cooperation also extends to the underwater medium. For example in servicing cases, with rendez-vous and docking aspects. In [16] platoons of underwater vehicles are considered

From the industrial point of view, it is interesting to notice that cooperative operational requirements may demand special working, maneuvering or dynamic positioning capabilities of the involved watercrafts.

\section{SCENARIOS AND INITLAL FORMALIZATION}

For the first steps of our research, an initial formalization of the cooperation scenarios has been introduced. The objective is to get a set of operation classes, with representative archetypes or examples for each class.

Let us begin by considering different types of things that can be present in an scenario:

- Passive and mute. For instance a rock, a wreck, an oil mouth, a barge.

- Passive, speaking. For instance, a buoy with a light or radio.

- Active, mute. For instance, A ship with a broken radio.

- Active and speaking. For instance, a ship with working radio.

This classification uses the active or passive terms, referring to auto-propulsion or not. The different things can act as agents, obstacles, targets, or beacons (references). The classification can be extended, considering, for instance, whether the things have any sensor (for instance, human vision, radar, etc.), and a decision system on board (for instance, a pilot). 
Having several watercrafts in cooperation, the decisions can be taken according with the following organization alternatives:

- A central coordinator. He takes the main decisions, which can be based on infornation from the team.

- Each individual, in silence. The individuals know what to do, only looking at the rest of the team. The operation is pre-planned.

- Speaking individuals. They give information about their awn decisions.

- Hierarchical structure. For instance a fleet of small fleets.

Along an operation the organization may change. This includes the roles in the team; for instance, the coordinator may change. Organizational transitions can be notified to the team; or not, since they may obey to a pre-defined strategy.

In general, part of a operation can be planned in advance, and part must adapt to changing circumstances.

The operations can be characterized by the existence of certain virtual or physical constraints. For instance, channels or confined waters, positional formation of the ships, physical links between them (for instance, when towing or making transfers).

According with the team working structure, in each moment, there are several communications schemes to choose:

- Centralized polling. For instance, with a coordinator.

- Turn-following. For instance, using tokens or time stamps.

- CSMA. For instance, with wireless ETHERNET.

- A strategy with priorities

In general, information can be given on-demand, or given by own initiative (event-driven). The information can be broadcasted, or sent to a particular address.

Figure 3 shows the kind of cooperant agent we are thinking to develop, for simulation and experimental research. It is of hybrid nature, combining continuous time and discrete-event. Operations will be decomposed into phases. The behaviour of the agent in each phase is governed by a particular automaton, selected from a pre-built set of automata. A top decision system takes into account the role assigned to the agent and the information from outside.



Fig. 3. Functional diagram of a cooperant agent.

\section{THE OIL SPILL OVER SCENARIO}

It was noticed, during the response to the Prestige disaster , that booms are usually carried by only two ships. This limits the capability of the confinement operation. However, it may happen that there are difficulties in the coordination of ships, even for only two.

Our attention was attracted by this case. Trying to know more about what happens, a simulation study was initiated.

In a first simplified analysis, three phases were distinguished in the operation. The next three figures show these phases, using the particular case of four ships handling the boom.

The target is an oil leak floating in a certain place. The leak must be confined, and, may be, carried to another place.

The first phase is leaving the harbour and towing the boom near the target. Resistance to motion should be minimized., and the distance between ships must be safe enough. Figure 4 shows an schematic view of this initial phase. The ships must advance with same speed and heading. It is good to know what ship is slower, to adapt the team speed to a reference, leaving some margin for individual adaptations.

Figure 5 shows the second phase. The fleet maneuvers, heading to the leak and deploying the boom. There is an optimization issue: to determine the best leak side for beginning the oil confinement. Mind the course transients to avoid breaking the boom. Ships in the middle have two links, port and starboard sides, with the neighbours; so they have more difficult decisions. It seems a good alternative to coordinate both ships in the middle, and then each of them coordinate its neighbour at the boom extreme.
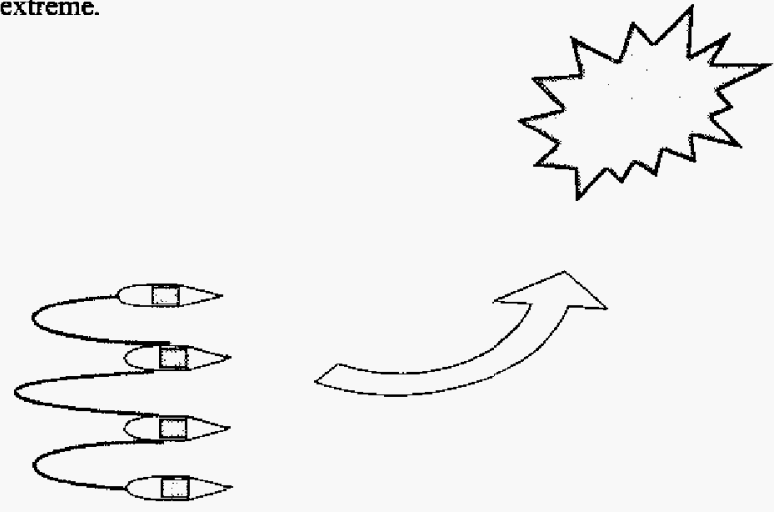

Fig. 4. First phase of the spill over operation.



Fig.5. Second phase of the spill over operation. 


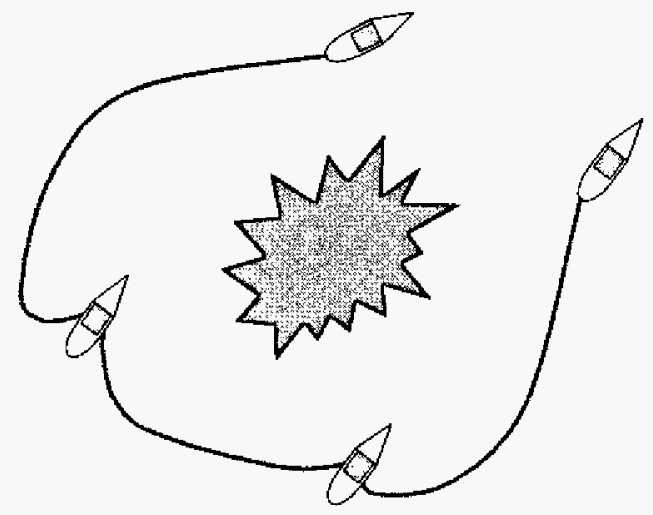

Fig.6. Third phase of the spill over operation.

The final phase is the leak confinement. The ships at the boom extremes should go faster than the other two ships. Four ships can form a sort of triangle for the confinement. Dynamic positioning may be required. At the end of this phase, a fourth boom can be fixed between the ships at the extremes, to close with a square the leak. Distances between ships may be relatively large, so it seems good to use GPS.

\section{MODELLING OF A SIMPLE CASE}

The description of the previous section is clearly qualitative. To enter into more details, the scenario was simplified and a first principles modelling was developed.

Consequently, the simple scenario considers two ships carrying a boom.

A first problem is to determine the forces and moments exerted by the boom on the ships, and the geometry of the boom along the system motion.

The second problem is that the boom makes the ships twist a little. If no beading control is applied to counteract this effect, the final state of the system is a tug of war between both ships.

Figure 7 shows the scenario. Two identical ships towing together a boom. The boom is made with identical rigid elements, joined by hinges.



Fig.7. Schematic view of the simple scenario.
Ships are described by their mass, mass inertia moment and three drag coefficients that represent the resistance to motion througb the fluid along surge, sway and yaw. Propulsion in surge direction is considered in terms of power. Steering action is also represented in terms of power to generate a moment in the yaw direction. The combined effect of propulsion power and steer power determines the ship course; both variables will be the input variables to be operated for control purposes.

Booms links are defined also by their mass, mass inertia moment and drag coefficients similar to those employed in the ships. Strains in the ends of the links complete the description for the boom dynamics.

The motion of a ship, in the $x$-y plane, can be described with the following equations:

$$
\begin{aligned}
& m_{b} a_{b x}= {\left[F_{m}-\mu_{i}\left(v_{b y} \operatorname{sen}(\theta)+v_{b x} \cos (\theta)\right)\right] \cos (\theta) } \\
&-\mu_{r} l_{s}\left(v_{b x} \operatorname{sen}(\theta)-v_{b y} \cos (\theta)\right) \operatorname{sen}(\theta) \\
& m_{b} a_{b y}= {\left[F_{m}-\mu_{l}\left(v_{b y} \operatorname{sen}(\theta)+v_{b x} \cos (\theta)\right)\right] \operatorname{sen}(\theta) } \\
&-\mu_{t} l_{s}\left(-v_{b x} \operatorname{sen}(\theta)+v_{b y} \cos (\theta)\right) \cos (\theta) \\
& M-\mu_{a} l_{s} \omega_{b}=I_{b} \alpha_{b}
\end{aligned}
$$

where $m b$ represents the mass of the ship, $a b x$ and $a b y$ represent the acceleration in axes $x$ and $y, F m$ is the surge force, $M$ is yaw moment, $\mu l ., \mu t, \mu a$ are the drag coefficients in surge, sway and yaw, $l s$ is the length of the ship, $l b$ is the moment of inertia.

The geometry of the ship's motion model is shown in figure 8 , where the angle $\theta$ represents the course, $v b$ is the ship's speed and $m c b$ is the position of the ship centre of mass. Figure 9 shows the geometry of the boom model.

The motion of the boom, which is attached to the ships, is deduced by first considering a link and then combining several links (imposing the corresponding closing conditions). The motion of a single generic link is given by:

$$
\bar{T}_{i, j+1}-\bar{T}_{i-1, i}-\frac{\left(\bar{v}_{i} \cdot \bar{n}_{i}|s+| \vec{v}_{i} \cdot\left(-\vec{p}_{i}\right) \mid q\right)}{\left|\vec{v}_{i}\right|} \vec{v}_{j}=m \vec{a}_{i}
$$

Were, $T$ represents the strain in a tip of the link, $n i$ is a normal vector, $p i$ is a vector opposite to the sense of the link, $q$ and $s$ represent longitudinal and perpendicular drag coefficients and $\mathrm{m}$ is the mass of the link.

$$
\left(\bar{T}_{i, i+1} \cdot \vec{n}_{i}\right) y+\left(\bar{T}_{i-1, i} \cdot \bar{n}_{i}\right) y-A \omega_{i}=I \alpha_{i}
$$

Were $l$ is half the length of the link, $A$ is a drag coefficient, and $I$ is the moment of inertia.

The closing condition is imposed by means of eq. (4.6)

$$
\bar{r}_{i}-l \bar{p}_{i}-\bar{l}_{i+1}-\bar{r}_{i+1}=0
$$

Were $r i$ is the position of the link. This equation is included to ensure boom continuity

\section{SIMULATIONS AND COMMENTS}

The figures in this section show superimposed "photographs" of the boom along time. The initial conditions have been forced to an extreme case with the boom almost straight, so the dynamics of the ships and the boom are bighly interrelated.

To avoid the tug of war, a simple proportional control, acting over the yaw moment, is put into action to force the ships to a fixed heading. Figure 10 shows the result. A transient appears at the beginning, sometimes driving the ships to separate and thus putting into risk the boom integrity.

To improve the control, a proportional control of the mutual distance has been added to the proportional course control of each ship. This implies a first issue of cooperation. The target for the distance control is to converge to less distance between ships, softening the boom tension. Figure 11 shows the result of the operation.

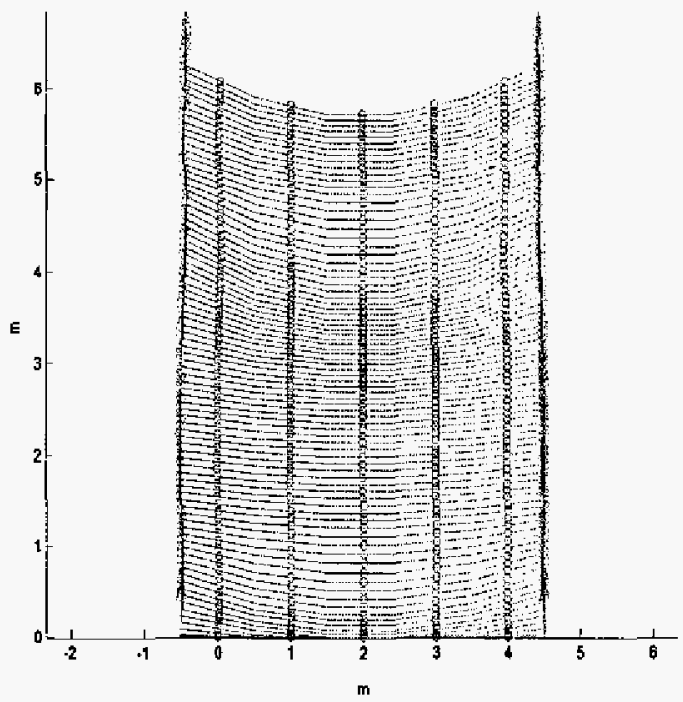

Fig, 10. Motion of the system with individual course control.

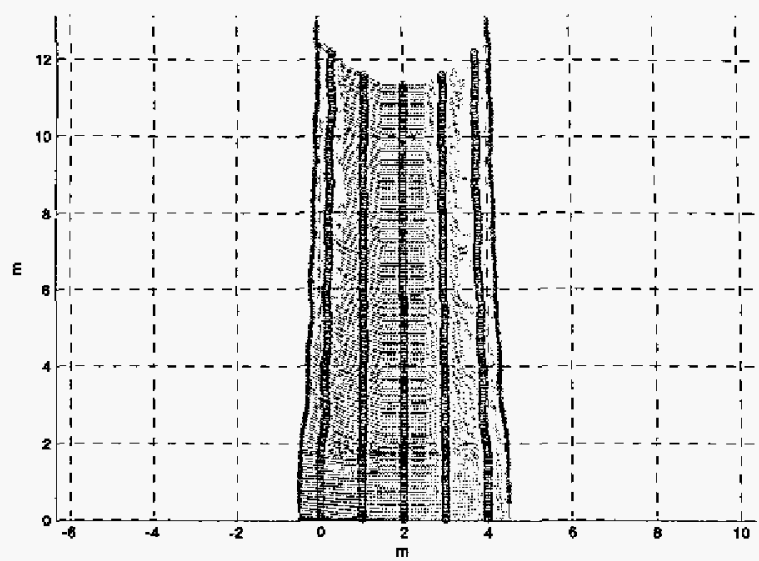

Fig.11. Motion of the system with individual course control and mutual distance control.

Figure 12 compares the horizontal component of the boom tension for three cases: no course control, individual course control, and the combined individual and mutual distance control.

Figure 13 shows the angle between the ship's attitude and the ship's velocity, for the last two cases. With individual control this angle has always the same sign, negative for one of the ships and positive for the other ship. Both ship stems are dragged inwards by the boom. With the combined control, the angles oscillate around cero.

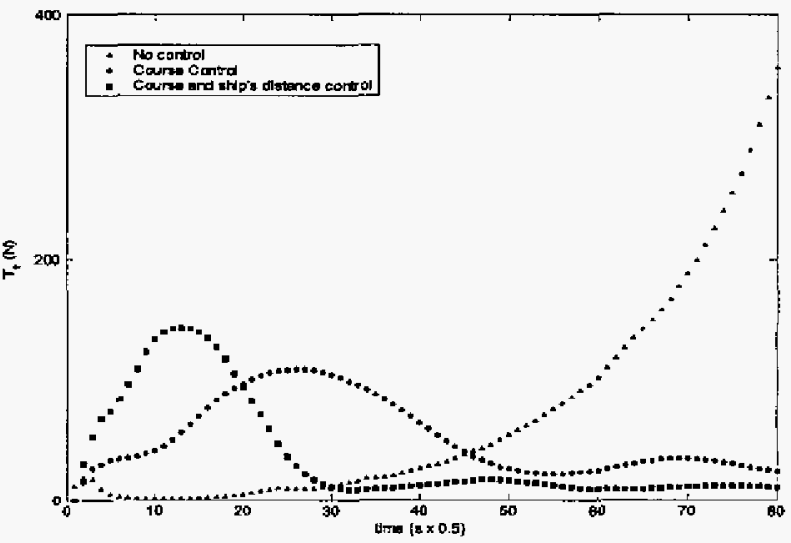

Fig.12. Boom tension for the three cases: no course control, individua course control, and combined individual and distance control

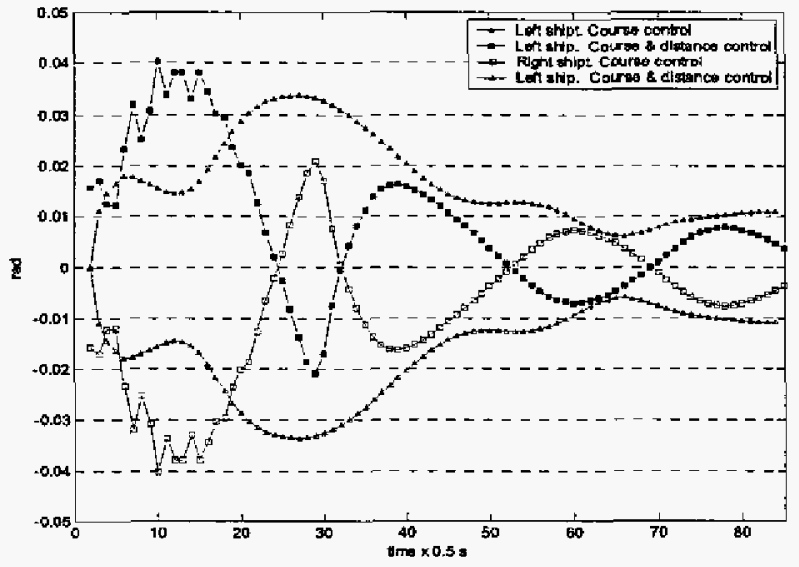

Fig.13. Angle between ships attitude and velocity for individual course control, and combined individual and distance control. 


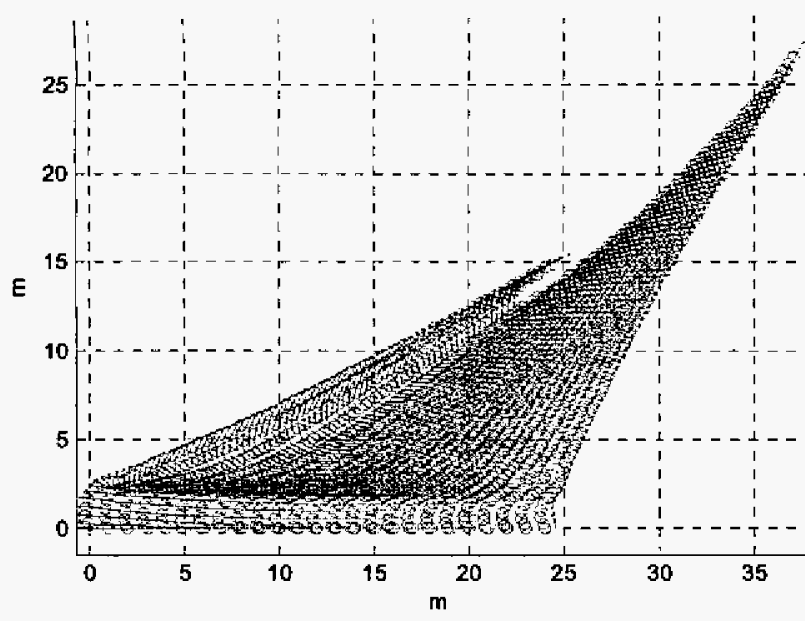

Fig.14. System tum, ships with same speed.

Figure 14 shows a $45^{\circ}$ right turn. Both ships at the same speed. It is clear that problems appear. A second cooperation issue rises: a change of speed is needed.

A speed control is added, in function of the angle between the desired course and a line linking both ships. The speed of the ships is increased or decreased until this angle is $90^{\circ}$.

Figure 15 shows the effect of this control strategy. Notice that the outer ship describes a wider curve due to sway at higher speed. It is advisable to have enough margin for the boom to straighten in certain moments of this maneuver.

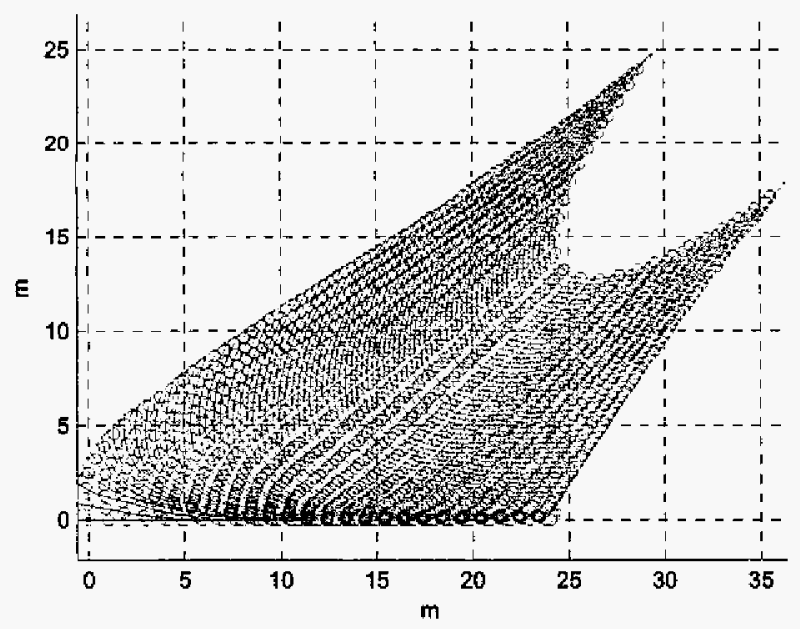

Fig.15. System turn with alignment control.

\section{vIII. CONCLUSIONS}

In this paper, a scenario for cooperative marine robotics has been introduced, including a brief review of the robotic context. Also, some mathematical modelling and initial formalization issues have been addressed.

The simple case of two ships towing a boom has been considered, in the initial steps of an spill over confinememt operation. Suggestive cooperation insights rapidly rise even at the beginning of the operation.

The research continues now considering the complete operation and other scenarios. More ships are being introduced.

First developments are made in Matlab. However att object-oriented approach is more opportune, and the use of $\mathrm{C}+$ is beginning now.
A team of scaled ships is under development. The on-board control includes an embedded $\mathrm{PC}$, with $\mathrm{C}$ code, positional sensors, speed and heading sensors, propeller control, and a radio-link to exchange messages with the other ships. The team will be used to test verbal protocols for cooperation control in scaled marine operations.

\section{Acknowledgments}

The authors would like to thank the Spanish MCYT, "Ministerio de Ciencia y Tecnologia", for their support (project DPL2000-0386-C03-02), the CEHIPAR staff for their cooperation, and IZAR for its recommendations.

\section{REFERENCES}

[1] J.M. Giron-Sierra, S. Esteban, B. De Andres, J.M. Diaz and J.M. Riola, "Experimental study of controlled flaps and T-foil for comfort imptovement of a fast ferry," in Proceedings IFAC Intl. Conf. Control Applications in Marine Systems CAMS2001, Glasgow, 2001.

[2] J.M. De la Cruz, J. Aranda, J.M. Giron-Sierra, F. Velasco, S. Esteban, J.M. Diaz, and B. De Andres, "Improving the comfort of a fast ferry with the control of flaps and T-foil," IEEE Control Systems Magazine, vol. 24, n. 2, pp. 47-60, April 2004.

[3] R. Brooks, "New approaches to robotics," Science, vol. 253, pp. 1227-32, September 1991.

[4] R.R. Murphy, Introduction to Al Robotics, MIT Press, 2000.

[5] J. Liu, and J. Wu, Mutti-Agent Robotic Systems, CRC Press, 2001.

[6] G. Weiss, Multiagent Systems, MIT Press, 1999.

[7] J.M. Giron-Sierra, C. Insaurralde, M. Seminario, J.F. Jimenez, J.A. Frutos, I. Perez, E. Buesa, and P. Klose, "Distributed control system for fuel management using canbus," in Proceedings 23th Digital Avionics Conference, Salt-Lake, 2004.

[8] U.M. Borghoff, and J.H. Schlichter, Computer Supported Cooperative Work, Springer, 2000.

[9] G.T. Tanner, J. Pappas, and V. Kumar, "Leader-to-formation stability," IEEE Trans. Robotics and Automation, vol. 20, n.3, pp. 443-455, June 2004.

[10] B. Barass, Ship Design and Performance for Masters and Mates, Butterworth-Heinemann, 2003.

[11] E. Kyrkjebo, and K.Y. Pettersen, "Ship replenishment using synchronization control, "“" in Proceedings IFAC MCMC 2003, Gerona, Spain, pp. 286-291, 2003.

[12] E. Kyrkjebo, M. Wondergem, K.Y. Pettersen and $\mathbf{H}$. Nijmeijer, "Experimental results on synchronization control of ship rendezvous operations," in Proceedings IFAC CAMS 2004, Ancona, Italy, pp. 453-458, 2004.

[13] H.M. Morishita, E.A. Tannuri, and T.T. Bravin, "Methodology for dynamic analysis of offloading operations," in Proceedings IFAC CAMS 2004, Ancona, Italy, pp. 459-464, 2004.

[14] D. Soetanto, L. Lapjerre and A. Pascoal, "Coordinated motion control of marine robots," in Proceedings IFAC MCMC2003, Gerona, Spain, pp. 250-255, 2003.

[15] V. Johansen, R. Skjetne, and A.J. Sorensen, "Maneuvering of towed interconnected marine systems," in Proceedings IFAC MCMC 2003, Gerona, Spain, pp. 292-297, 2003.

[16] D.J. Stilwell, and B.E. Bishop, "Platoons of underwater vehicles," IEEE Cotrol Systens Magazine, vol. 20, pp. 45-52, December 2000. 